Research Article

\title{
Electrical Conductivity-Based Estimation of Unfrozen Water Content in Saturated Saline Frozen Sand
}

\author{
Zejin Lai $\mathbb{D}^{1},{ }^{1}$ Xiaodong Zhao $\mathbb{D}^{1}{ }^{1}$ Rui Tang, ${ }^{1}$ and Jinhong Yang ${ }^{2}$ \\ ${ }^{1}$ State Key Laboratory for Geomechanics and Deep Underground Engineering, China University of Mining and Technology, \\ Xuzhou 221116, China \\ ${ }^{2}$ China Coal Construction Group Limited Corporation, Beijing 102218, China
}

Correspondence should be addressed to Zejin Lai; dalcin@163.com and Xiaodong Zhao; zxdcumt@126.com

Received 26 September 2020; Revised 13 January 2021; Accepted 19 January 2021; Published 4 February 2021

Academic Editor: Gang Zhou

Copyright (c) 2021 Zejin Lai et al. This is an open access article distributed under the Creative Commons Attribution License, which permits unrestricted use, distribution, and reproduction in any medium, provided the original work is properly cited.

\begin{abstract}
The salinity of the pore solution is closely associated with the unfrozen water content and can be reflected by variation in electrical conductivity in frozen soils. However, the influence of salinity was not considered in the existing models for estimation of unfrozen water content based on electrical conductivity measurement, and a model considering the effect of salt content was therefore developed to estimate the change of unfrozen water content of saline sands with variation of salt content $(0 \%, 0.2 \%$, and $1 \%)$. The unfrozen water content and the electrical resistivity were measured by nuclear magnetic resonance (NRM) and using resistance test equipment under a temperature ranging from $25^{\circ} \mathrm{C}$ to $-15^{\circ} \mathrm{C}$, respectively. The results indicated that the model using a cementation exponent expressed by a piecewise function with respect to temperature can produce a reasonable estimation on the content of unfrozen water. There was an essential difference between nonsaline and saline frozen sands in the increase of electrical resistivity due to the different reduction rates of unfrozen water content. The variation of electrical resistivity in nonsaline sand was mainly caused by the decrease of free water when temperature was higher than the freezing point and adsorbed water when temperature was lower than the freezing point, whereas the reduction of free water in two stages was the main reason for the variation of electrical resistivity in saline sand. The results and data obtained provided a basis for further developing a novel approach to measure the unfrozen water content in the field.
\end{abstract}

\section{Introduction}

The unfrozen water content in frozen soils has significant influences on mechanical properties and frost heave characteristics that also closely involve the stability analysis of engineering in cold regions [1-3]. Therefore, a series of methods to measure the content of unfrozen water in frozen soils were used, such as the nuclear magnetic resonance (NMR) method [4, 5], time-domain reflectometry (TDR) method [6-8], and differential scanning calorimetry (DCC) method $[9,10]$. Nevertheless, the above methods were mainly applied to a small-scaled specimen in laboratory experiments. For monitoring the distribution of unfrozen water or ice content in large-scaled outdoor sites, many scholars used a convenient electrical resistivity test and stated that electrical resistivity followed a semilogarithmic relationship with unfrozen water content and a power law with ice content [11-14]. Meanwhile, theoretical models of the electrical resistivity in frozen soils were constantly updated. Shan et al. deduced a model that considered three conductive paths (soil particles, ice-water mixtures, and soil-ice-water mixtures) [15], and Tang et al. proposed four types of the microunit with the variation of unfrozen water content [16]. Although the mineral composition in frozen soils is complex, the salinity is generally low. In fact, the saline frozen soil produced by freezing-thawing action and evaporation was widely distributed in permafrost regions [17]. The impacts of salinity caused the decline of the freezing point and significant variation in unfrozen water content and also the electrical resistivity of the pore solution $[18,19]$.

In conclusion, previous studies mainly focused on the electrical resistivity of frozen soils without salt. The research 
on electrical resistivity of saline frozen soils was lacking. In order to study the effect of unfrozen water content on the mechanical behavior of saline frozen soil, it is necessary to estimate the unfrozen water content in saline frozen soil. In the present study, the influences of temperature, salt content, and unfrozen water content on electrical conductivity were considered, and then, a new model capable of estimating the unfrozen water content in saturated cohesionless soils with different salt contents based on Archie's law during freezing was established. Additionally, the unfrozen water content and the electrical resistivity with various salt contents were determined. Finally, the rationality of this model was checked and verified by detailed comparisons with the measured datum from NMR. Then, the effects of temperature, unfrozen water content, and concentration on electrical resistivity were analyzed.

\section{Theoretical Basis for Frozen Soil Electrical Conductivity}

2.1. Effect of Water Content. According to Archie's law [20], the electrical conductivity of unsaturated cohesionless soil can be expressed as follows:

$$
\sigma_{T_{r}, s p, c_{0}}=\theta_{T_{r}}^{m} \sigma_{T_{r}, w, c_{0}} S^{n}
$$

where $\sigma_{T_{r}, s p, c_{0}}$ and $\sigma_{T_{r}, w, c_{0}}$ are, respectively, the electrical conductivities of the unsaturated cohesionless soil and the pore solution with salt concentration $c_{0}$ at the reference temperature equal to $T_{r}, S$ is the degree of saturation, $n$ is the saturation coefficient, $m$ represents the cementation exponent, and $\theta_{T_{r}}$ is the volumetric water content at temperature equal to $T_{r}$.

During the freezing process, the electrical conductivities of ice and air were all far smaller than that of the pore water solution [21, 22], thus $S$ in equation (1) was considered as the relative unfrozen water content. Furthermore, $m$ depended upon the change of porosity and connectivity [23-26]. The porosity gradually reduced due to the ice formation, which changed the connectivity of the pores. Therefore, $m$ should be a function of temperature in frozen soils, that was to say $m=m(T)$. So, equation (1) can be rewritten as follows:

$$
\sigma_{T, s p, c_{0}}=\theta_{T}^{m(T)} \sigma_{T, w, c_{0}} S^{n},
$$

where $\sigma_{T, s p, c_{0}}$ and $\sigma_{T, w, c_{0}}$ are the electrical conductivities of frozen soils and the pore solution with salt concentration $c_{0}$ at the temperature $T$, respectively and $\theta_{T}$ is the volumetric water content at temperature equal to $T$.

2.2. Effect of Temperature. The dynamic viscosity coefficient of the solution decreased with the increase in temperature, which was beneficial to ion migration within the solution. Therefore, electrical conductivity of the solution increased with the temperature. Campbell et al. built a relationship between electrical conductivity of the solution and temperature based on experimental data [27]:

$$
\sigma_{T, w, c_{0}}=\sigma_{T_{r}, w, c_{0}}\left[1+\alpha\left(T-T_{r}\right)\right],
$$

where $\alpha$ is the temperature coefficient, and $\alpha$ was generally estimated as 0.021 or 0.0187 [27, 28].

Inserting equation (3) into equation (2), the following expression can be obtained:

$$
\sigma_{T, s p, c_{0}}=\theta_{T}^{m(T)} \sigma_{T_{r}, w, c_{0}}\left[1+\alpha\left(T-T_{r}\right)\right] S^{n},
$$

2.3. Effect of Salt Concentration. Upon freezing, Konrad and Mccammon stated that the solutes were rejected from the pore ice [29], which increased the concentration of the pore solution. And, the electrical conductivity of the pore solution changed linearly with respect to the saturation in a frozen state [30]. However, Weiss et al. obtained a nonlinear relationship between electrical conductivity and concentration [31] based on the theory by Snyder [32], so the expression can be written in the following form:

$$
\sigma \propto \frac{I}{1+G \sqrt{I}}
$$

where $G$ is the electrical parameter, $0.4(\mathrm{~mol} / \mathrm{L})^{-0.5}$, for a typical pore solution [31] and $I$ is the ionic strength of the pore solution, which is determined by species with charge numbers Zand molar concentration $c$, and it is defined as follows:

$$
I=\frac{1}{2} \sum_{i=1}^{N} Z_{i}^{2} c_{i} .
$$

The concentration of the pore solution might be changed substantially due to salt crystallization (the reduction of solute content) and ice formation (the reduction of the solvent). If the effect of solute crystallization was considered, the variation in electrical conductivity of the pore solution can be proposed as follows:

$$
\frac{\sigma_{T_{r}, w, c_{1}}}{\sigma_{T_{r}, w, c_{0}}}=\frac{I_{1}}{I_{0}} \frac{1+G \sqrt{I_{0}}}{1+G \sqrt{I_{1}}},
$$

where $\sigma_{T_{r}, w, c_{1}}$ is electrical conductivity of the pore solution with salt concentration $c_{1}$ at the reference temperature $T_{r}, I_{0}$, and $I_{1}$ are the ionic strength of the pore solution before and after solute crystallization.

Similarly, the concentration of the pore solution gradually increased owing to solvent reduction during the drying process, which was inversely proportional to saturation. Thus, based on equation (5), the ratio of electrical conductivity of the pore solution before and after reduction of the solvent can be given as follows [31]:

$$
\frac{\sigma_{T_{r}, w, c_{2}}}{\sigma_{T_{r}, w, c_{0}}}=\frac{I_{2}}{I_{0}} \frac{1+G \sqrt{I_{0}}}{1+G \sqrt{I_{2}}} \approx \mathrm{S}^{\delta-1},
$$

where $\sigma_{T_{r}, w, c_{2}}$ is electrical conductivity of the pore solution with salt concentration $c_{2}$ at the reference temperature $T_{r}, I_{2}$ is ionic strength of the pore solution after the reduction of the solvent, and $\delta$ is the fitting coefficient determined by direct measurement of the pore fluid ionic strength. Generally, $\delta=0.0565 \ln \left(I_{s}\right)+0.213$ and $I_{s}$ is ionic strength in a saturated sample [33]. The solution freezing process was 
similar to the drying process, which was commonly characterized by solvent reduction and solute concentration increase, namely, the variation of soil pore solution concentration in the frozen state can be described by equation (8).

\section{Theoretical Model considering Salinity}

The electrical conductivity is primarily affected by temperature, water content, and pore solution concentration. The soil electrical conductivity successively undergoes two stages during freezing. Initially, solute solubility varies with temperature when the soil temperature is higher than the freezing point, which probably induces variation in pore solution concentration due to solute precipitation. The water content does not change under an unfrozen state. Thus, the soil electrical conductivity is affected by temperature and pore solution concentration. Considering equations (4) and (7), the variation of soil electrical conductivity due to pore solution concentration and temperature can be obtained:

$$
\frac{\sigma_{T, s s, c_{1}}}{\sigma_{T_{r}, s s, c_{0}}}=\frac{\sigma_{T, s s, c_{1}}}{\sigma_{T_{r}, s s, c_{1}}} \frac{\sigma_{T_{r}, s s, c_{1}}}{\sigma_{T_{r}, s s, c_{0}}}=\left[1+\alpha\left(T-T_{r}\right)\right] \frac{I_{1}}{I_{0}} \frac{1+G \sqrt{I_{0}}}{1+G \sqrt{I_{1}}},
$$

where $\sigma_{T, s s, c_{1}}$ is the electrical conductivity of soil under an unfrozen state with pore solution concentration $c_{1}$ at temperature $T$. With regard to the sodium chloride solution, the ionic strength of the pore solution is identical with salt concentration based on equation (6). Hence, the variation of pore solution concentration can be deduced as follows according to equation (9):

$$
\left\{\begin{array}{l}
c_{1}=\left(\frac{A G+\sqrt{A^{2} G^{2}+4 A}}{2}\right)^{2}, \\
A=\frac{\sigma_{T, s s, c_{1}}}{\sigma_{T_{r}, s s, c_{0}}} \frac{1}{1+\alpha\left(T-T_{r}\right)} \frac{c_{0}}{1+G \sqrt{c_{0}}},
\end{array}\right.
$$

Afterward, the soil temperature continued to decline and was lower than the freezing point, which caused a decrease in the unfrozen water content due to formation of ice crystal. The pore solution concentration increased with the decrease of unfrozen water content and satisfied equation (8). So, the variation of soil electrical conductivity was influenced by temperature, unfrozen water content, and pore solution concentration. Combining equations (4)-(8), the ratio of soil electrical conductivity in stage with temperature lower than the freezing point to the initial soil electrical conductivity can be given as follows:

$$
\begin{aligned}
\frac{\sigma_{T, s p, c_{2}}}{\sigma_{T_{r}, s s, c_{0}}}=\frac{\sigma_{T, s p, c_{2}}}{\sigma_{T_{r}, s s, c_{1}}} \frac{\sigma_{T_{r}, s s, c_{1}}}{\sigma_{T_{r}, s s, c_{0}}}=\frac{\sigma_{T_{r}, s p, c_{2}}}{\sigma_{T_{r}, s s, c_{1}}} \frac{\theta_{T}^{m(T)}}{\theta_{T_{r}}^{m\left(T_{r}\right)}} \\
\\
\cdot\left[1+\alpha\left(T-T_{r}\right)\right] \frac{I_{1}}{I_{0}} \frac{1+G \sqrt{I_{0}}}{1+G \sqrt{I_{1}}}, \\
=S^{n} \frac{I_{2}}{I_{1}} \frac{1+G \sqrt{I_{1}}}{1+G \sqrt{I_{2}}} \frac{\theta_{T}^{m(T)}}{\theta_{T_{r}}^{m\left(T_{r}\right)}}\left[1+\alpha\left(T-T_{r}\right)\right] \frac{I_{1}}{I_{0}} \frac{1+G \sqrt{I_{0}}}{1+G \sqrt{I_{1}}},
\end{aligned}
$$

where $\sigma_{T, s p, c_{2}}$ is electrical conductivity of soil under a frozen state with pore solution concentration $c_{2}$ at temperature $T$. It is noted that $I_{0}$ is replaced by $I_{1}$ if equation (8) was used, so the term $I_{2} / I_{1} 1+G \sqrt{I_{1}} / 1+G \sqrt{I_{2}} \approx S^{\delta-1}$. Equation (11) can be rewritten in the following form:

$$
\frac{\sigma_{T, s p, c_{2}}}{\sigma_{T_{r}, s s, c_{0}}}=S^{\delta-1+n} \frac{\theta_{T}^{m(T)}}{\theta_{T_{r}}^{m\left(T_{r}\right)}}\left[1+\alpha\left(T-T_{r}\right)\right] \frac{I_{1}}{I_{0}} \frac{1+G \sqrt{I_{0}}}{1+G \sqrt{I_{1}}} .
$$

Solving equation (12), then the unfrozen water content is defined by

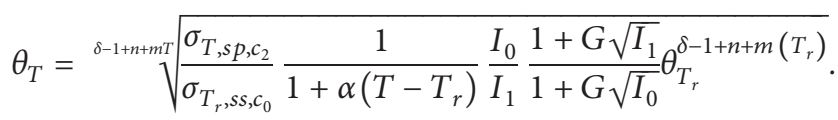

According to equation (13), the unfrozen water content in stage (II) can be calculated only with the normalized soil electrical conductivity from the measured datum. Meanwhile, by combining equation (13) and equation (8), the pore solution concentration in stage with temperature lower than the freezing point can be obtained as follows:

$$
\left\{\begin{array}{l}
c_{2}=\left(\frac{\mathrm{BG}+\sqrt{B^{2} G^{2}+4 B}}{2}\right)^{2}, \\
B=S^{\delta-1} \frac{c_{1}}{1+G \sqrt{c_{1}}} .
\end{array}\right.
$$

\section{Test Apparatus, Materials, and Methods}

4.1. Test Apparatus. Two-electrode and four-electrode were the main methods for measuring soil electrical resistivity [34]. The four-phase electrode method affected the internal structure of soil, especially the small-scaled specimens. In order to simplify the test operation, a two-phase electrode method was adopted in this study. A schematic diagram of the frozen and unfrozen soil electrical resistivity test device is shown in Figure 1.

The test system consisted of two modules: a temperature control system and an electrical resistivity measuring system. The temperature ranged from $-30^{\circ} \mathrm{C}$ to $+40^{\circ} \mathrm{C}$ with an accuracy of $\pm 0.05^{\circ} \mathrm{C}$. The soil temperature was measured by thermocouples with an interval of $1.5 \mathrm{~cm}$ along the specimen height in order to ensure a uniformity in temperature distribution. The soil electrical resistivity was measured via Data Taker 85G. The conductive copper paste was evenly smeared on the bottom and upper surfaces of the soil sample. Then, two copper electrodes were used to reduce the influence of contact resistance. The copper electrodes and wire were welded to ensure the stability of the test.

The unfrozen water content of frozen soils was measured by NMR, as shown in Figure 2. The test apparatus was divided into four parts, an industrial computer for data storage, processing, and sets of boundary temperatures ranging from $-20^{\circ} \mathrm{C}$ to $+30^{\circ} \mathrm{C}$ with an accuracy of $\pm 0.01^{\circ} \mathrm{C}$, a magnet unit that provided a uniform and stable main 


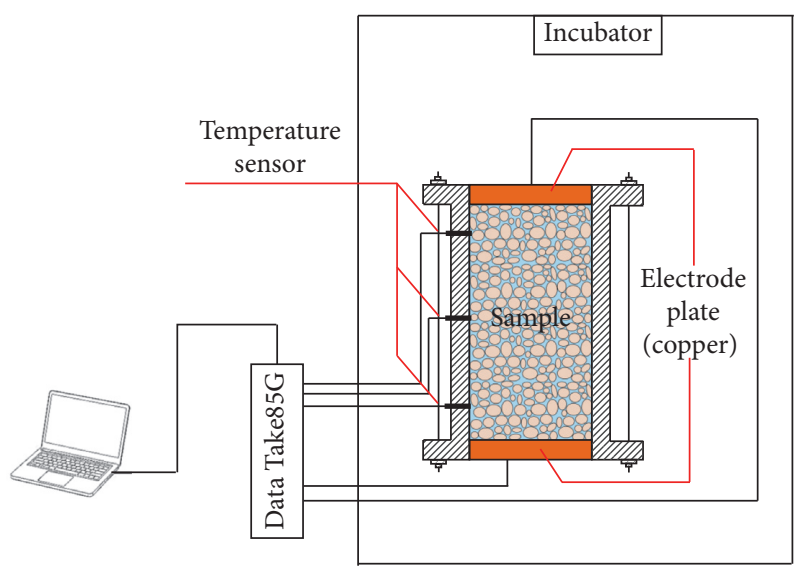

FIgURE 1: The electrical conductivity test system.

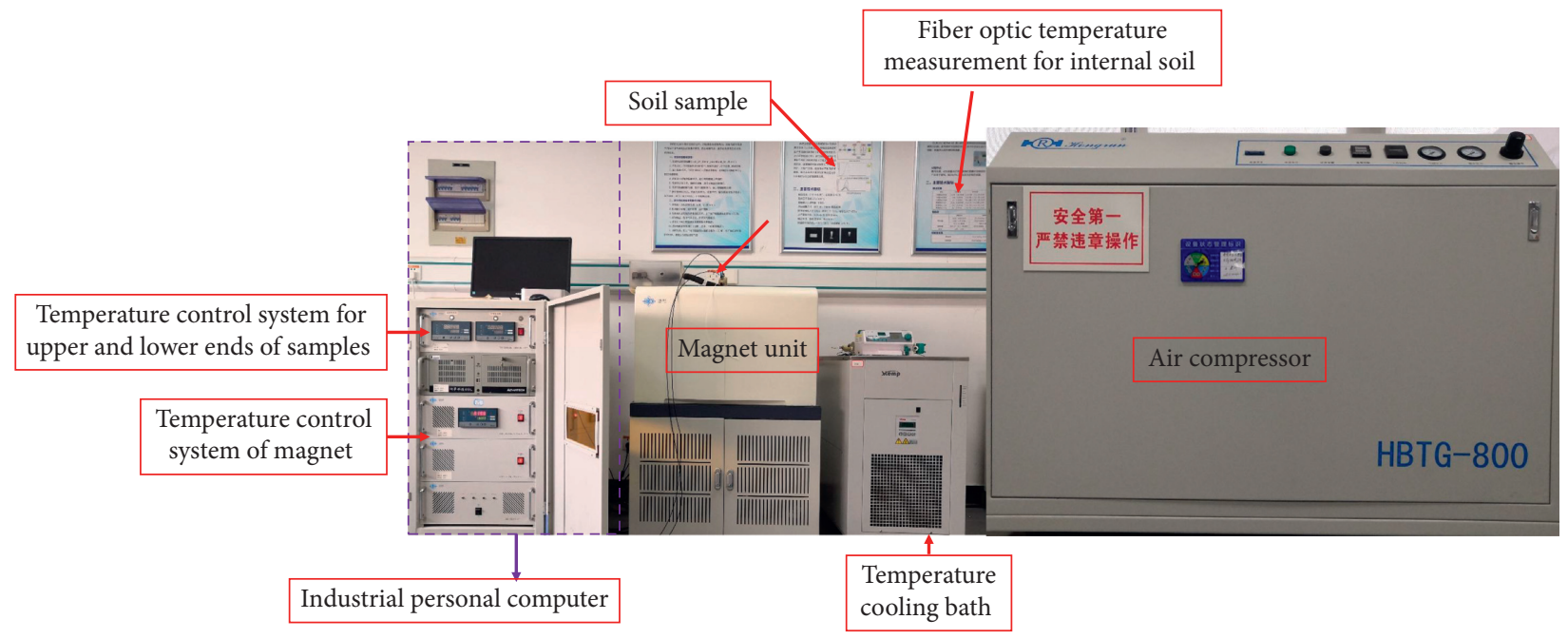

Figure 2: The unfrozen water content test system.

magnetic field with an intensity of $0.5 \mathrm{~T}$ at a temperature of $32 \pm 0.01^{\circ} \mathrm{C}$, an air compressor that provided dry air for cooling, and a bath that turned dry gas into low-temperature gas.

4.2. Sample Preparation. The material was sand with particle size ranging from 0.5 to $1.0 \mathrm{~mm}$. In order to remove the interference of original salinity in soil, the sand was washed using deionized water with an electrical conductivity $\leq 0.25 \mu \mathrm{S} / \mathrm{m}$ and dried in a stove. Then, the nonsaline sand was poured into a cylindrical mold (a height of $6 \mathrm{~cm}$ and a diameter of $5.15 \mathrm{~cm}$ for measuring electrical resistivity and a height of $5 \mathrm{~cm}$ and a diameter of $2.5 \mathrm{~cm}$ for measuring unfrozen water content) to prepare the soil sample with a target dry density of $1.63 \mathrm{~g} / \mathrm{m}^{3}$. Afterwards, these soil samples were saturated by the solution under a vacuum of about $100 \mathrm{kPa}$. The measured saturation was $24.54 \%$.

4.3. Test Scheme. In terms of the report about the salt content of natural soil [35], a salt content of $0.2 \%$ was designed in the test. Chlorine saline soil was selected, and the chlorine salt was provided by sodium chloride with a purity higher than $99.8 \%$. The salt concentration equal to the mass ratio of salt to soil particle was designed in three levels of $0 \%$, $0.2 \%$, and $1 \%$, respectively. The temperature range was from $+25^{\circ} \mathrm{C}$ to $-15^{\circ} \mathrm{C}$ considering the natural temperature change in the site.

4.4. Test Procedure. The soils sample with three temperature sensors was put into the incubator. The temperature of the incubator was set at $+25^{\circ} \mathrm{C}$, and it was maintained for 15 minutes. Then, the resistance of the soil sample was recorded. The resistivity was calculated using equation (15). Afterward, the temperature in the incubator was decreased to the next target value, and the above operation was repeated until the soil temperature reached $-15^{\circ} \mathrm{C}$.

$$
\rho=\frac{R \times S}{L},
$$

where $R$ is resistance of the tested soil sample, whose unit is $\Omega, S$ is the cross-sectional area, whose unit is $\mathrm{m}^{2}$, and $L$ is the height of the soil sample, whose unit is $\mathrm{m}$. 
In the NMR test, the air compressor and bath were opened to ensure a smooth gas path when the soil sample was put into the test chamber. The temperatures of the upper and bottom ends of the soil sample remained at $+25^{\circ} \mathrm{C}$. The temperature within the soil sample was collected by the optical fiber sensor with 60 seconds interval. The nuclear magnetic signals were gathered when the temperature within the soil sample reached $+25^{\circ} \mathrm{C}$. Afterward, the temperature of the upper and bottom ends of the soil sample decreased to the next target value, and the above operation was repeated until the soil temperature reached $-15^{\circ} \mathrm{C}$.

\section{Results and Discussion}

5.1. Temperature Coefficient. Equation (3) showed that the temperature coefficient was an important parameter in determining the variation of resistivity. The curve slope of 0.0175 close to 0.0187 proposed by Hayley [28] in Figure 3 was the temperature coefficient concerned in equation (4).

5.2. Electrical Conductivity. The electrical response of the soil sample with different salinities varied with temperature in the cooling process and is shown in Figure 4.

As shown in Figure 4, the electrical resistivity under various salt contents presented similar variation characteristics. The electrical resistivity increased with the decrease in temperature and decreased with the increase in salt content under an identical temperature because the increasing charge concentration caused the soil to be more conductive. Inflection points were observed in the electrical resistivity curve, which represented the initial freezing temperature. According to the descriptions by Konrad and Mccammon [29], the initial freezing temperatures of soil should be $-0.5^{\circ} \mathrm{C}$ and $-2.601^{\circ} \mathrm{C}$ corresponding to a salt content of $0.2 \%$ and $1 \%$, respectively, which were consistent with temperatures occurring at inflection points. The soil electrical resistivity increased slightly with the decrease in the temperature under an unfrozen state. However, under a frozen state, the soil electrical resistivity increased rapidly with the decrease in the temperature, and the increasing rate was much higher than that under an unfrozen state due to the reduction of unfrozen water content [15]. Figure 4(b) shows the variation of normalized electrical conductivity with temperatures ranging from $+25^{\circ} \mathrm{C}$ to $-15^{\circ} \mathrm{C}$. The normalized conductivity of soil under different salt contents tended to be consistent under an unfrozen state, which implied that the temperature coefficient of 0.0175 can describe the influence of temperature on the electrical resistivity of saline sand.

The variation of electrical resistivity with temperature under different salt content during freezing are shown in Figure 5 . The electrical resistivity increased slowly with the decrease in the temperature when the temperature was higher than the freezing point and then increased rapidly when the temperature exceeded the freezing point. The soil sample with a salt content of $0,0.2$, and $1 \%$ has an initial freezing temperature of $-1.5^{\circ} \mathrm{C},-3^{\circ} \mathrm{C}$, and $-8.5^{\circ} \mathrm{C}$,

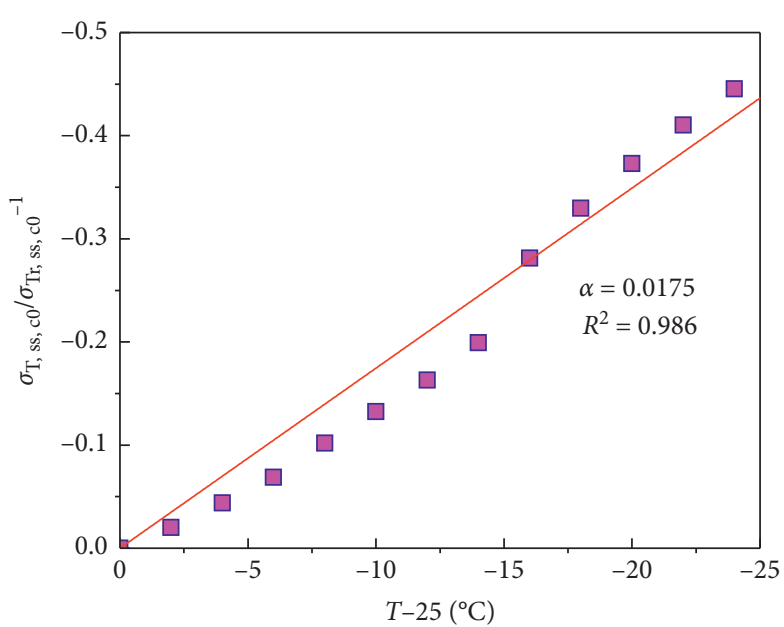

FIgURE 3: Temperature coefficient.

respectively. Obviously, the threshold extended as the salt content increased. On the one hand, salt inhibited the increase in electrical resistance, and a greater concentration caused a more obvious inhibition effect [28]. On the other hand, the unfrozen water content increased with the increase in salt content at an identical temperature due to the depression of the freezing point, which slowed down the increase in electrical resistivity.

The variations of electrical resistivity and the changing rate under different salt contents with unfrozen water content are shown in Figure 6. The soil electrical resistivity increased gradually with the decrease in the unfrozen water content due to the obstruction of the current flow path deriving from the continuous transformation of pore water into pore ice. However, the soil electrical resistivity slightly increased, and the unfrozen water content was nearly unchanged under an unfrozen state. By calculating the changing rate under a freezing state, the first point at which the gradient began to change was defined as an inflection point. Thus, the variation of electrical resistivity under different salt contents with unfrozen water content can be divided into two stages based on the changing rate.

Figure 6(a) represents the variation of nonsaline sand electrical resistivity. In the first stage before the inflection point, the unfrozen water content dropped sharply to around $7 \%$ when the soil temperature declined from 0 to $-2.5^{\circ} \mathrm{C}$, but the changing rate of soil electrical resistivity increased relatively slow. Afterward, although the unfrozen water content slowly reduced to approximately $6 \%$, the changing rate of soil electrical resistivity was significantly accelerated, whereas the three stages of electrical resistivity were not observed in nonsaline sand under a frozen state as described by Tang et al. [16]. This might be associated with the differences existing in particle size distribution and the nonconductivity of sand particles.

Figures 6(b) and 6(c), respectively, show that the variations of the sand electrical resistivity and the changing rate with unfrozen water content at $0.2 \%$ and $1 \%$ salt content. Because of the nonconductivity of sand particles, the saline sand exhibited two conducting paths: the free water path and 


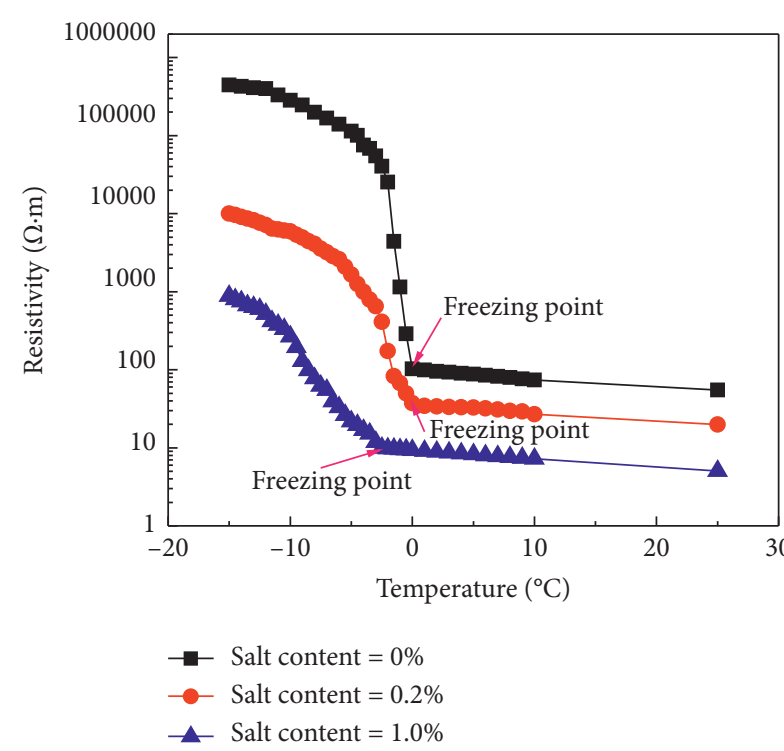

(a)

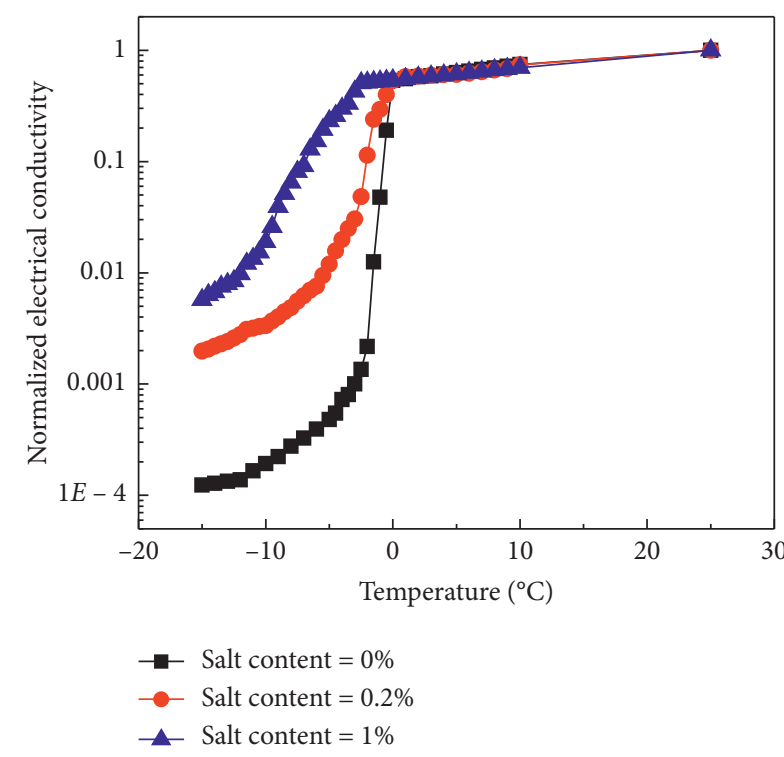

(b)

FIGURE 4: Electrical response during freezing. (a) Electrical resistivity. (b) Electrical conductivity.

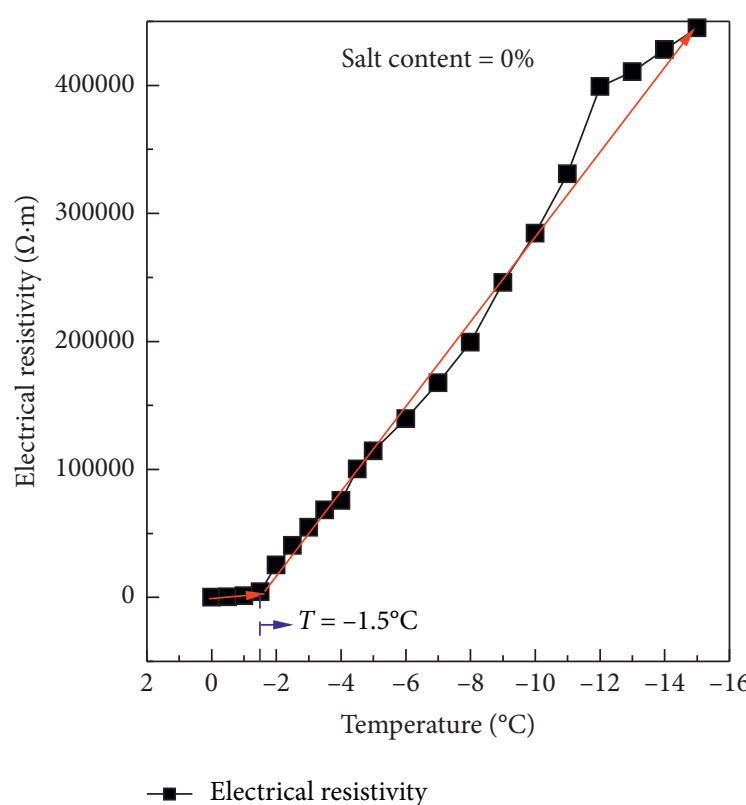

(a)

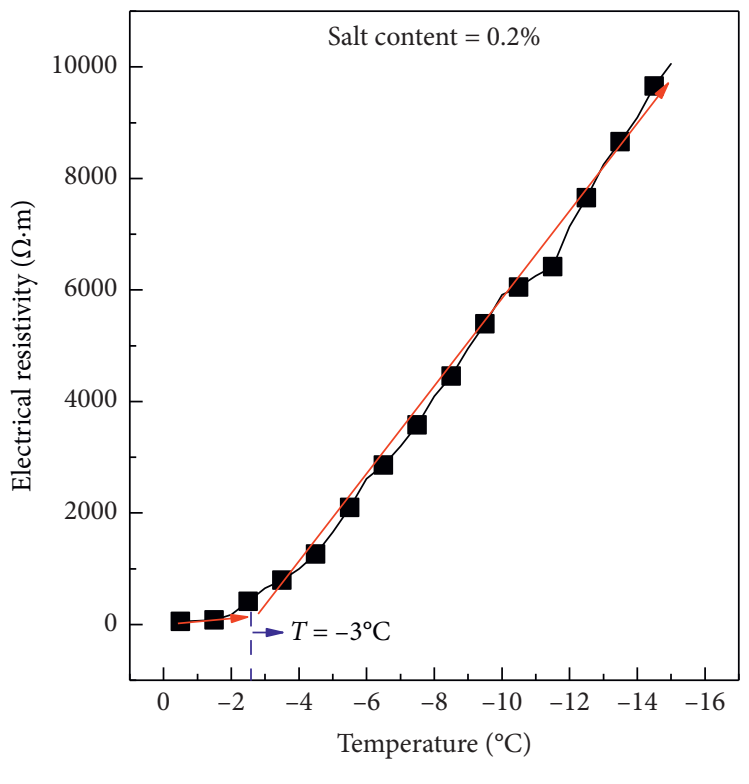

- Electrical resistivity

(b)

Figure 5: Continued. 


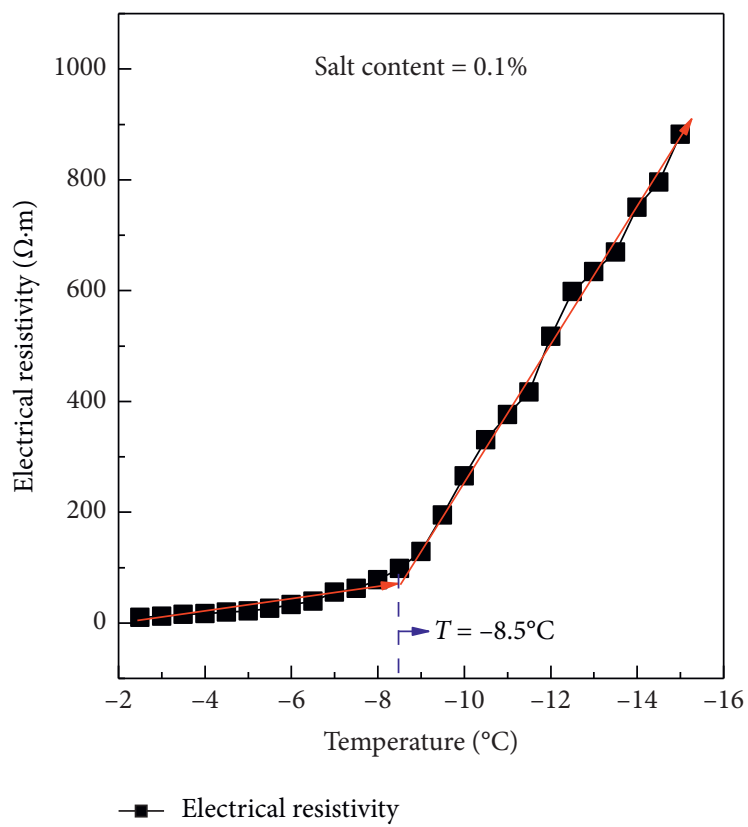

(c)

FIGURE 5: Electrical resistivity varies with temperature under a freezing state. (a) Without salinity. (b) Salinity of $0.2 \%$. (c) Salinity of $1.0 \%$.

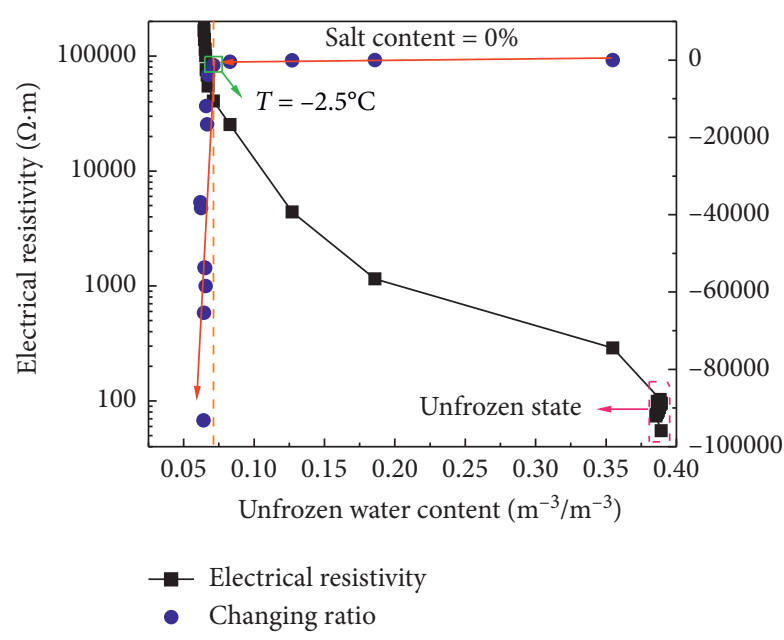

(a)

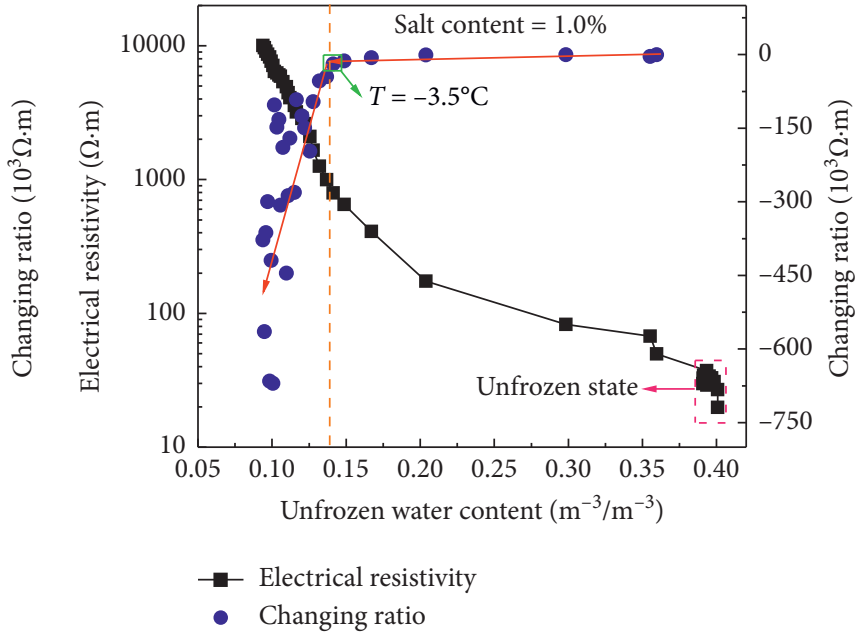

(b)

FIgUre 6: Continued. 


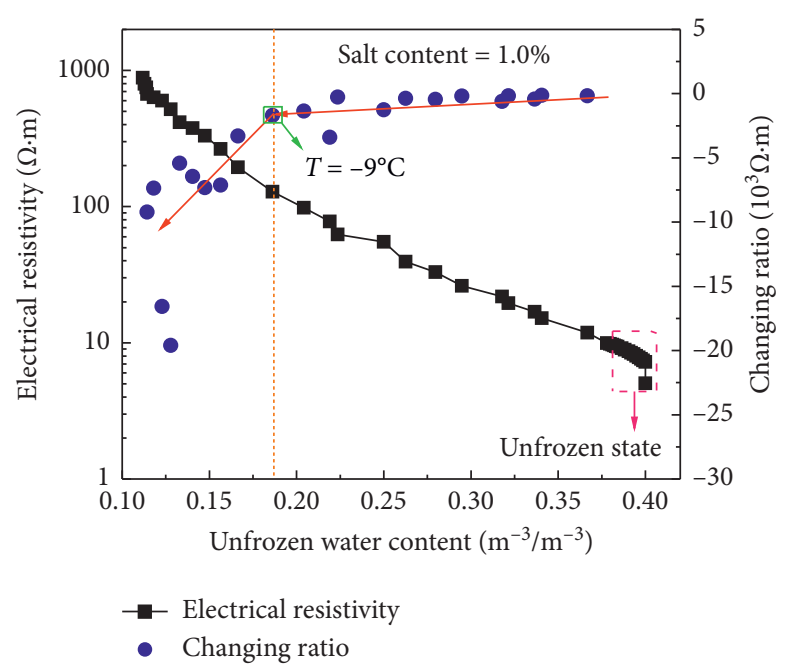

(c)

FIGURE 6: Electrical resistivity and the changing ratio of electrical resistivity varied with unfrozen water content under a freezing state. (a) Without salinity. (b) Salinity of $0.2 \%$. (c) Salinity of $1.0 \%$.

water film path existing at the particle surface. By comparing Figures $6(\mathrm{a})-6(\mathrm{c})$, although the changing rates of the sand electrical resistivity with different salt contents had similar variation characteristics, they behaved significantly different with orders of magnitude. Further, the unfrozen water content corresponding to the inflection points were different with a value of $7 \%$ for nonsaline, $12 \%$ for $0.2 \%$ salt content, and $18 \%$ for $1 \%$ salt content. These deviations can be interpreted from the changes in unfrozen water content. For nonsaline sand, there were two conducting paths before the inflection point, and the increase in electrical resistivity at this stage was due to the ice blocking. In the next stage after the inflection point, the free water was substantially frozen. So, the adsorbed water film was the conducting path, and a thinner of the unfrozen water film caused a more tortuous of the conducting path. As stated, a directional moving rate of conductive particles in such a path decreased, and the soil resistivity therefore increased. However, the unfrozen water content corresponding to inflection points in saline sand $(0.2 \%$ and $1 \%$ salt contents) were much higher than that in nonsaline sand. Therefore, the variation of the saline sand electrical resistivity was only related to the reduction of free water.

5.3. Estimation of Unfrozen Water Content. The determination of the saturation exponent and the cementation exponent was needed. Archie has pointed out that the saturation exponent was two for both the consolidated and unconsolidated sand [20]. For the cementation exponent, Friedman stated that there was a physical explanation for the saturation exponent larger than the cementation exponent [21]. The water film around solid particles became thinner, and the conducting path became tortuous when the saturation degree decreased. Moreover, the cementation exponent of sand was $1.495<2$ according to the test results by Chen et al. [36]. In the present study, two methods for determining the cementation exponent were considered. One method was that the cementation coefficient was constant to 1.495 and remained constant with temperature. The cementation exponent as a piecewise function of temperature was considered in the other method based on the study by Glover [23]. For convenience, the cementation exponent was assumed as 1.495 in an unfrozen state, and it increased linearly from 1.495 to 2 between the freezing temperature and $-10^{\circ} \mathrm{C}$ and maintained at 2 when the soil temperature was lower than $-10^{\circ} \mathrm{C}$. Therefore, the variation of unfrozen water content with temperature can be estimated by combining the measured electrical resistivity and equation (15), as shown in Figure 7.

As seen from Figure 7, the variation characteristic of the unfrozen water content estimated by the model with the temperature was consistent with the measured data, which was applicable for frozen nonsaline sand and frozen saline sand. However, there was a difference between the estimated unfrozen water content using two methods, and the difference was larger as the temperature decreased. The prediction efficiency from the model utilizing the latter method was more reasonable. It verified Glover's view on new interpretation of the cementation exponent that it was sensitive to changing connectivity of the sensitivity and the changing porosity of the connectedness. In other words, the cementation exponent gradually increased during freezing due to ice formation. However, the prediction efficiency became worse with the increase in the salt content due to the uncertainty of the parameters.

5.4. Estimation of Salt Concentration. The salt concentration variations can be found in Figure 8. The pore solution concentration was changed due to solute crystallization based on changes in solubility from the reference temperature to the initial freezing temperature. However, the 


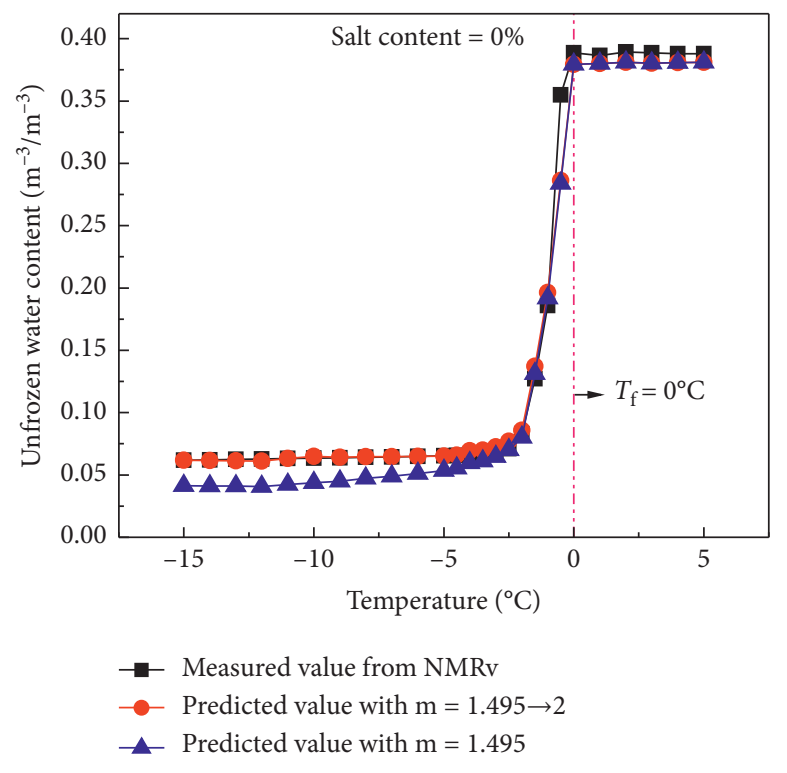

(a)

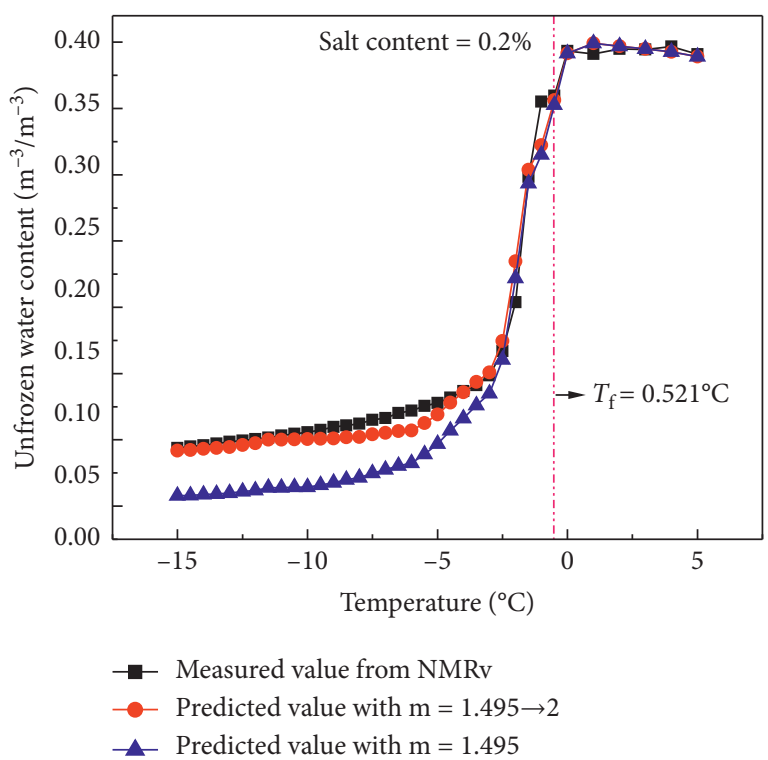

(b)

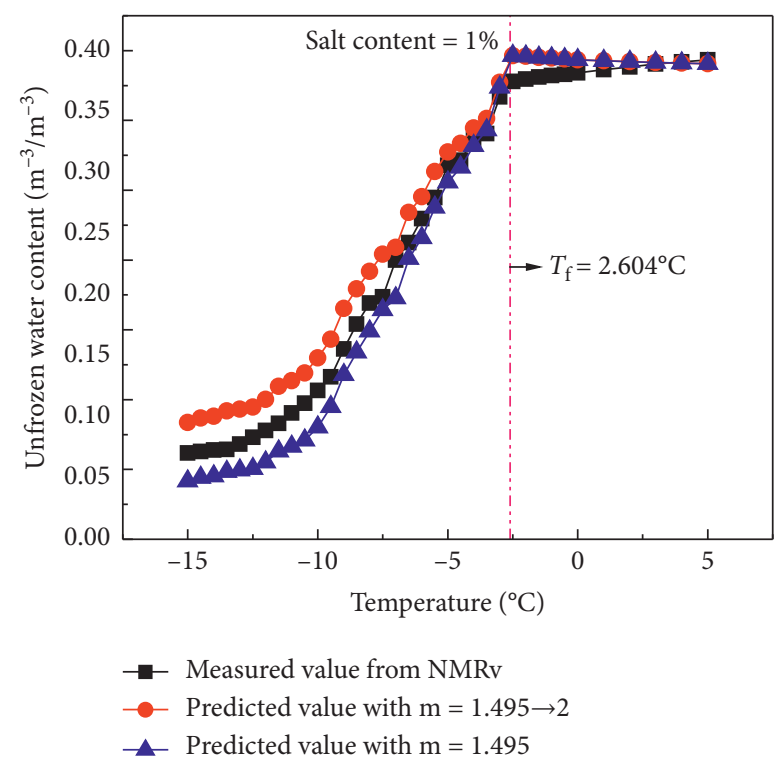

(c)

FIgURE 7: Comparisons between measured and predicted unfrozen water contents. (a) Without salinity. (b) Salinity of $0.2 \%$. (c) Salinity of $1.0 \%$.

concentration did not substantially change due to the high solubility of sodium chloride, which satisfied the phase diagram Then, soil temperature further decreased and ice crystals were formed. Therefore, the pore solution concentration gradually increased due to the decrease in the unfrozen water content.

5.5. Multiplication Factor. According to equations (9) and (12), the electrical resistivity was induced by various factors and was multiplied by each other. Therefore, the temperature, the unfrozen water content, and the salt concentration multiplication factors were defined, respectively:

$$
\begin{aligned}
& f(T)=\left[1+\alpha\left(T-T_{r}\right)\right], \\
& f(\theta)=S^{n} \frac{\theta_{T}^{m(T)}}{\theta_{T_{r}}^{m\left(T_{r}\right)}} \\
& f(c)=\frac{I_{1}}{I_{0}} \frac{1+G \sqrt{I_{0}}}{1+G \sqrt{I_{1}}}
\end{aligned}
$$

where $f(T), f(\theta)$, and $f(c)$ represent the temperature, unfrozen water content, and concentration multiplication factor, respectively. The multiplication factor has "dilution" or inhibition influence on the electrical resistivity if it was 


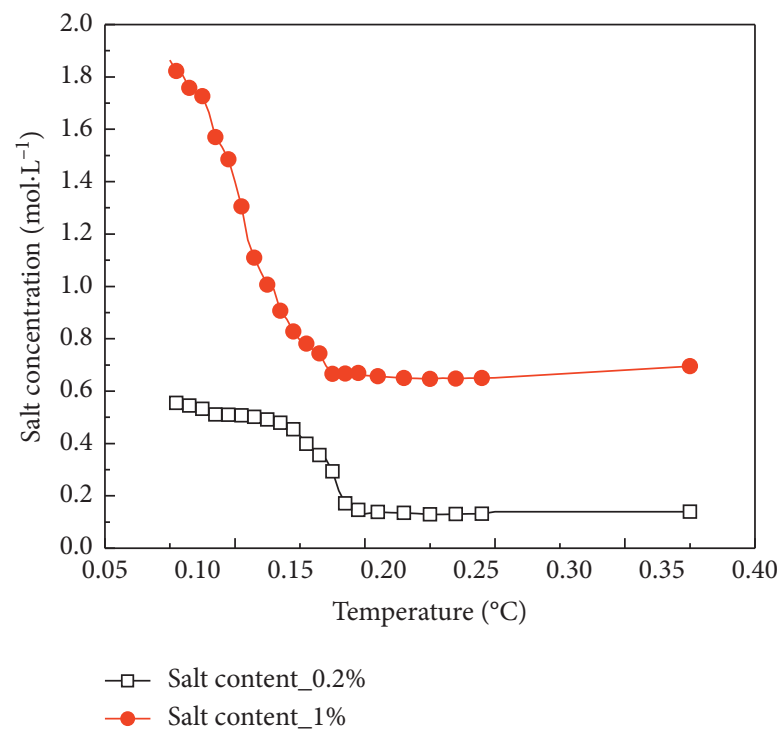

Figure 8: The variation of pore salt concentration during freezing.
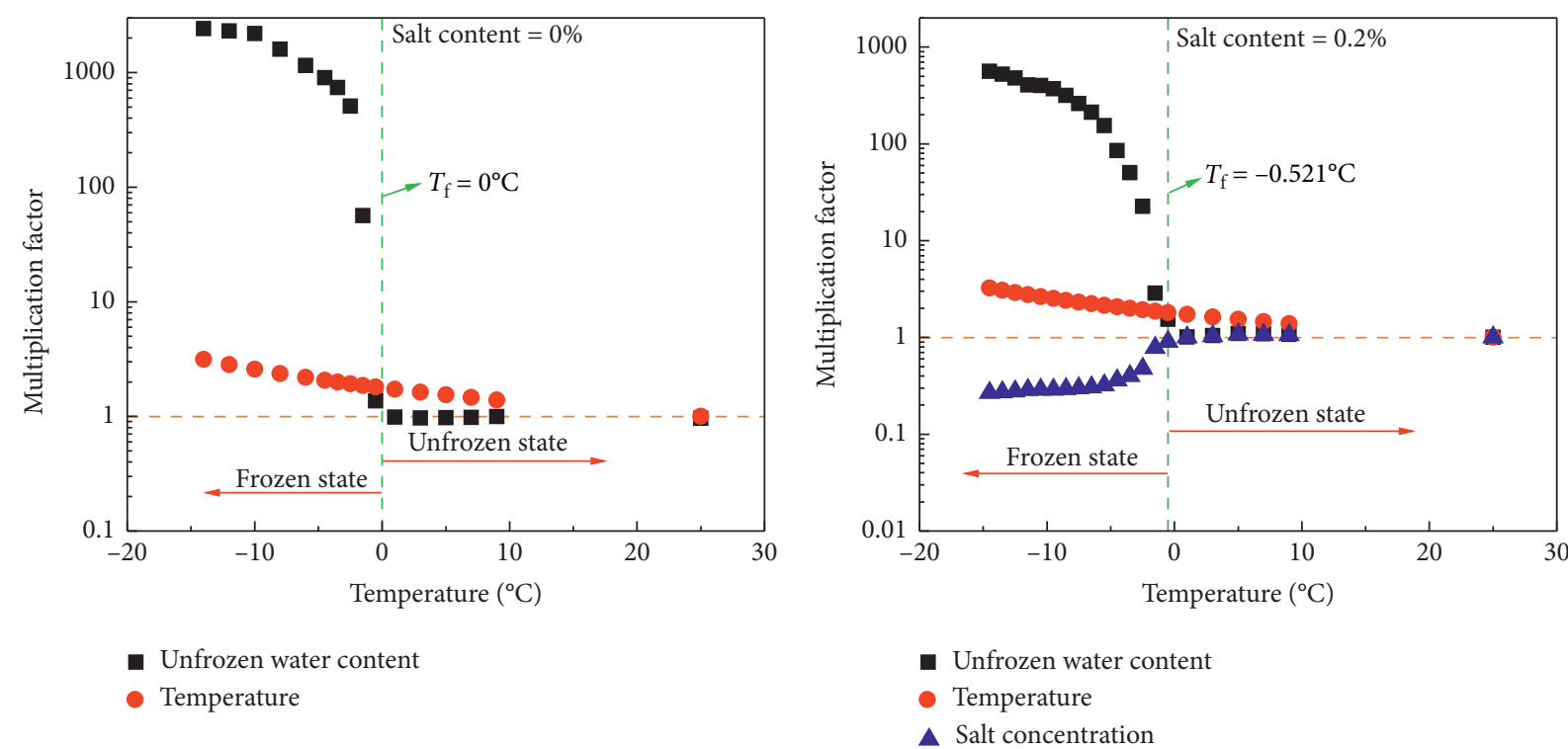

(a)

(b)

Figure 9: Continued. 


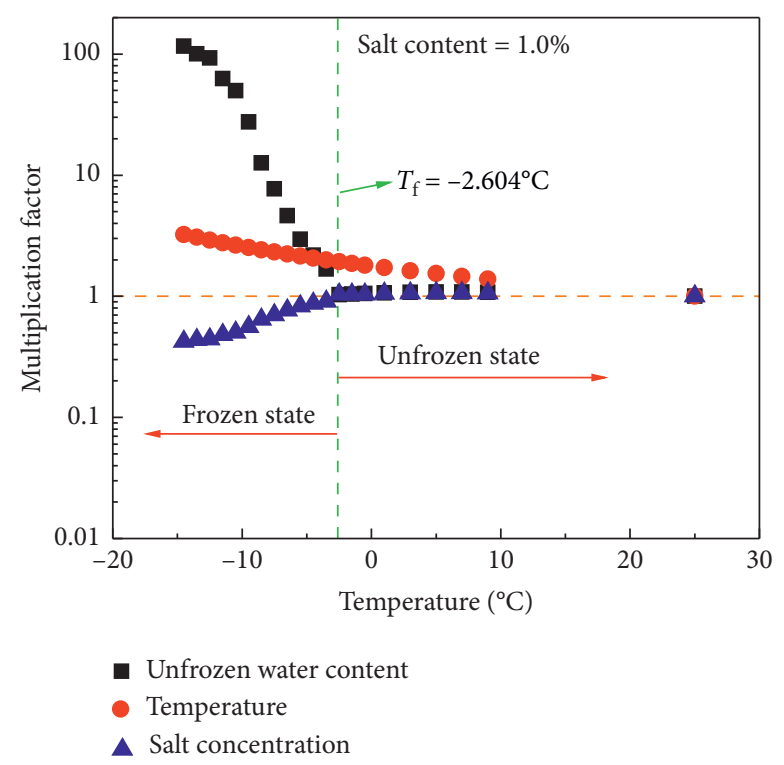

(c)

FIGURE 9: The variation of multiplication factors with temperature during freezing. (a) Without salinity. (b) Salinity of $0.2 \%$. (c) Salinity of $1.0 \%$.

less than 1, has no influence if it equaled 1, and has "enrichment" or promotion influence if it was greater than 1.

In Figure 9, the temperature multiplication factor increased linearly with the decrease of temperature in the cooling process. The unfrozen water content and the concentration multiplication factors had different characteristics before and after soil freezing. The unfrozen water content multiplication factor was maintained at one before freezing temperature, but it increased nonlinearly with the decrease of temperature after freezing temperature. Further, the unfrozen water content multiplication factor was larger than the temperature multiplication factor. The concentration multiplication factor of saline sand shown in Figures 9(b) and 9(c) maintained at one before freezing temperature and gradually decreased with the decrease of temperature after freezing temperature, which indicated that the concentration factor had an inhibitory effect on the electrical resistivity.

\section{Conclusions}

In this study, a model considering the effects of salt, temperature, and water content on soil resistivity was established to estimate the variation of unfrozen water and the pore concentration in the cooling process. The electrical resistivity and the unfrozen water content of the sand with different salinities were tested. Based on the experimental results and model analysis, the following conclusions can be drawn:

(1) The model can reflect the variation of unfrozen water content and the pore concentration in saline sand during the freezing process. It provides a theoretical basis for further research on the characteristics of frost heave in saline permafrost. The cementation exponent should be used as a function of temperature, which is capable of estimating a more reasonable result. However, there might be uncertainty using the model combined with conductivity data to estimate the content of unfrozen water.

(2) During the freezing process, the variations of electrical resistivity in nonsaline sand and saline sand with the unfrozen water content can be divided into two stages with a threshold of freezing temperature. For nonsaline sand, the freezing of free water led to the increase of electrical resistivity in the first stage, and the decrease of adsorbed water at the surface of particles led to the increase of resistivity in the next stage. However, for saline sand, the decrease of free water was the main reason for the increase of the electrical resistivity in two stages. The free water was completely frozen, and the adsorbed water decreased as the continuing decline in temperature, so there was a significantly faster increase in electrical resistivity.

(3) The electrical resistivity was affected by the temperature, unfrozen water content, and salt concentration, and three multiplication factors were defined. In the frozen state, the temperature and the unfrozen water content multiplication factors promoted the increase of the electrical resistivity. Particularly, the unfrozen water content multiplication factor was the main promoting factor, but the concentration multiplication factor generated negative feedback.

\section{Data Availability}

The data used to support the findings of this study are available from the corresponding author upon request. 


\section{Conflicts of Interest}

The authors declare that they have no conflicts of interest regarding the publication of this paper.

\section{Acknowledgments}

This work was supported by the National Natural Science Foundation of China (Grant nos. 51304209, 41271096, and 41772338), 111 Project (Grant no. B14021), and Open Fund of State Key Laboratory of Frozen Soil Engineering (Grant no. SKLFSE201704).

\section{References}

[1] G. Zhou, K. Hu, X. Zhao, J. Wang, H. Liang, and G. Lu, "Laboratory investigation on tensile strength characteristics of warm frozen soils," Cold Regions Science and Technology, vol. 113, pp. 81-90, 2015.

[2] X. Xu, Y. Wang, R. Bai, C. Fan, and S. Hua, "Comparative studies on mechanical behavior of frozen natural saline silty sand and frozen desalted silty sand," Cold Regions Science and Technology, vol. 132, pp. 81-88, 2016.

[3] X. Xu, Q. Li, Y. Lai, W. Pang, and R. Zhang, "Effect of moisture content on mechanical and damage behavior of frozen loess under triaxial condition along with different confining pressures," Cold Regions Science and Technology, vol. 157, pp. 110-118, 2019.

[4] A. M. Kruse and M. M. Darrow, "Adsorbed cation effects on unfrozen water in fine-grained frozen soil measured using pulsed nuclear magnetic resonance," Cold Regions Science and Technology, vol. 142, pp. 42-54, 2017.

[5] K. Yoshikawa and P. P. Overduin, "Comparing unfrozen water content measurements of frozen soil using recently developed commercial sensors," Cold Regions Science and Technology, vol. 42, no. 3, pp. 250-256, 2005.

[6] Z. Tian, T. Ren, Y. Kojima, Y. Lu, R. Horton, and J. L. Heitman, "An improved thermo-time domain reflectometry method for determination of ice contents in partially frozen soils," Journal of Hydrology, vol. 555, pp. 786-796, 2017.

[7] D. E. Patterson and M. W. Smith, "The measurement of unfrozen water content by time domain reflectometry: results from laboratory tests," Canadian Geotechnical Journal, vol. 18, no. 1, pp. 131-144, 1981.

[8] M. Stähli and D. Stadler, "Measurement of water and solute dynamics in freezing soil columns with time domain reflectometry," Journal of Hydrology, vol. 195, no. 1-4, pp. 352-369, 1997.

[9] M. Koniorczyk and P. Konca, "Kinetics of water freezing in mesopores determined by differential scanning calorimetry," International Journal of Thermal Sciences, vol. 122, pp. 124132, 2017.

[10] T. Kozlowski, "A simple method of obtaining the soil freezing point depression, the unfrozen water content and the pore size distribution curves from the DSC peak maximum temperature," Cold Regions Science and Technology, vol. 122, pp. 18$25,2016$.

[11] G. A. Oldenborger and A.-M. LeBlanc, "Monitoring changes in unfrozen water content with electrical resistivity surveys in cold continuous permafrost," Geophysical Journal International, vol. 215, no. 2, pp. 965-977, 2018.
[12] C. Hauck, M. Böttcher, and H. Maurer, "A new model for estimating subsurface ice content based on combined electrical and seismic data sets," The Cryosphere, vol. 5, no. 2, pp. 453-468, 2011.

[13] B. Dafflon, S. Hubbard, C. Ulrich et al., "Geophysical estimation of shallow permafrost distribution and properties in an ice-wedge polygon-dominated Arctic tundra region," Geophysics, vol. 81, no. 1, pp. WA247-WA263, 2016.

[14] R. Fortier, A.-M. LeBlanc, M. Allard, S. Buteau, and F. Calmels, "Internal structure and conditions of permafrost mounds at Umiujaq in Nunavik, Canada, inferred from field investigation and electrical resistivity tomography," Canadian Journal of Earth Sciences, vol. 45, no. 3, pp. 367-387, 2008.

[15] W. Shan, Y. Liu, Z. Hu, and J. Xiao, "A model for the electrical resistivity of frozen soils and an experimental verification of the model," Cold Regions Science and Technology, vol. 119, pp. 75-83, 2015.

[16] R. Tang, G. Zhou, W. Jiao, and Y. Ji, "Theoretical model of hydraulic conductivity for frozen saline/non-saline soil based on freezing characteristic curve," Cold Regions Science and Technology, vol. 165, Article ID 102794, 2019.

[17] G. Li, W. Yu, W. Ma et al., "Experimental study of characteristics of frost and salt heaves of saline highway foundation soils in seasonally frozen regions in Gansu Province," Rock and Soil Mechanics, vol. 30, no. 8, pp. 2276-2280, 2009, in Chinese.

[18] H. Bing and W. Ma, "Laboratory investigation of the freezing point of saline soil," Cold Regions Science and Technology, vol. 67, no. 1-2, pp. 79-88, 2011.

[19] A. Banin and D. M. Anderson, "Effects of salt concentration changes during freezing on the unfrozen water content of porous materials," Water Resources Research, vol. 10, no. 1, pp. 124-128, 1974.

[20] G. E. Archie, "The electrical resistivity log as an aid in determining some reservoir characteristics," Transactions of the AIME, vol. 146, no. 1, pp. 54-62, 1942.

[21] S. P. Friedman, "Soil properties influencing apparent electrical conductivity: a review," Computers and Electronics in Agriculture, vol. 46, no. 1-3, pp. 45-70, 2005.

[22] V. F. Petrenko, "Electrical properties of ice," Journal of Applied Meteorology, vol. 10, p. 81, 1993.

[23] P. Glover, "What is the cementation exponent? A new interpretation," The Leading Edge, vol. 28, no. 1, pp. 82-85, 2009.

[24] W. Han, G. Zhou, D. Gao et al., "Experimental analysis of the pore structure and fractal characteristics of different metamorphic coal based on mercury intrusion-nitrogen adsorption porosimetry," Powder Technology, vol. 362, pp. 386-398, 2020.

[25] W. Han, G. Zhou, Q. Zhang, H. Pan, and D. Liu, "Experimental study on modification of physicochemical characteristics of acidified coal by surfactants and ionic liquids," Fuel, vol. 266, Article ID 116966, 2020.

[26] W. Han, G. Zhou, M. Xing et al., "Experimental investigation on physicochemical characteristics of coal treated with synthetic sodium salicylate-imidazole ionic liquids," Journal of Molecular Liquids, Article ID 114822, 2020.

[27] R. B. Campbell, C. A. Bower, and L. A. Richards, "Change of electrical conductivity with temperature and the relation of osmotic pressure to electrical conductivity and ion concentration for soil extracts," Soil Science Society of America Journal, vol. 13, no. C, pp. 66-69, 1949.

[28] K. Hayley, L. R. Bentley, and M. Gharibi, "Time-lapse electrical resistivity monitoring of salt-affected soil and groundwater," Water Resources Research, vol. 45, no. 7, 2009. 
[29] J.-M. Konrad and A. W. Mccammon, "Solute partitioning in freezing soils," Canadian Geotechnical Journal, vol. 27, no. 6, pp. 726-736, 1990.

[30] E. G. Hivon and D. C. Sego, "Strength of frozen saline soils," Canadian Geotechnical Journal, vol. 32, no. 2, pp. 336-354, 1995.

[31] J. Weiss, K. Snyder, J. Bullard, and D. Bentz, "Using a saturation function to interpret the electrical properties of partially saturated concrete," Journal of Materials in Civil Engineering, vol. 25, no. 8, pp. 1097-1106, 2013.

[32] K. A. Snyder, C. Ferraris, N. S. Martys, and E. J. Garboczi, "Using impedance spectroscopy to assess the viability of the rapid chloride test for determining concrete conductivity," Journal of Research of the National Institute of Standards and Technology, vol. 105, no. 4, pp. 497-509, 2000.

[33] Y. Wang, F. Gong, D. Zhang, and T. Ueda, "Estimation of ice formation in mortar saturated with sodium chloride solutions," Construction and Building Materials, vol. 144, pp. 238-251, 2017.

[34] Y. Chu, S. Liu, B. Bate, and L. Xu, "Evaluation on expansive performance of the expansive soil using electrical responses," Journal of Applied Geophysics, vol. 148, pp. 265-271, 2018.

[35] D. Wu, Y. Lai, Q. Ma, and C. Wang, "Model test study of water and salt migration and deformation characteristics in seasonally frozen soil," Rock and Soil Mechanics, vol. 37, no. 2, pp. 465-476, 2016, in Chinese.

[36] R. Chen, W. Chen, J. Wang, and Y. Chen, "Electrical conductivity of pore water in saturated sand and its measurement technology," Chinese Journal of Geotechnical Engineering, vol. 32, no. 5, pp. 780-783, 2010, in Chinese. 